\title{
IRECIST Partial Response
}

National Cancer Institute

\section{Source}

National Cancer Institute. iRECIST Partial Response. NCI Thesaurus. Code C142358.

A finding indicating that there is a decrease based on a predetermined threshold in the size and the extent of tissue involvement by cancer following an initial apparent radiographic progression. 